\title{
Tumor carcinoide bronquial en pediatría: reporte de caso
}

Fecha de recepción: 10/05/2016 | Fecha de aprobación: 18/10/2016 | 08/08/2016

\author{
Melissa de Castro Hernández ${ }^{1}$ \\ Pontificia Universidad Javeriana, Colombia \\ Ángela María Soler ${ }^{2}$ \\ Pontificia Universidad Javeriana, Colombia \\ Juan S. Arjona ${ }^{3}$ \\ Pontificia Universidad Javeriana, Colombia
}

1 Médica pediatra, Hospital Universitario San Ignacio-Pontificia Universidad Javeriana, Bogotá, Colombia

2 Médica pediatra, Hospital Universitario San Ignacio-Pontificia Universidad Javeriana, Bogotá, Colombia.

3 Médico general, Pontificia Universidad Javeriana, Bogotá, Colombia.

Cómo citar: De Castro Hernández M, Soler ÁM, Arjona JS. Tumor carcinoide bronquial en pediatría: reporte de caso. Univ Med. 2017;58(1): 1-5. https://doi.or g/10.11144/Javeriana.umed58-1.tcbp

\section{RESUMEN}

Los tumores primarios de pulmón son poco frecuentes en el paciente pediátrico. Dentro de ellos, el tumor carcinoide bronquial es el más común. Clínicamente, su manifestación más usual es como infección respiratoria a repetición; sin embargo, su presentación puede ser variada. Reportamos el caso de una paciente de catorce años de edad con clínica de dolor torácico y síntomas respiratorios.

Palabras clave

tumor carcinoide; tumor pulmonar primario; pediatría.

\begin{abstract}
Lung primary tumors are bizarre in pediatrics. Among them, carcinoid bronchial tumor is the most common. The classical manifestation is recurrent respiratory tract infection, but the clinical presentation can be variable. We report the case of a 14 years-old patient with chest pain and respiratory symptoms.
\end{abstract}

Keywords

carcinoid tumor; primary lung tumor; pediatrics.

\section{Reporte de caso}

El caso corresponde a una adolescente de catorce años de edad con el antecedente de un episodio broncobstructivo a los trece años manejado con broncodilatador y simpatectomía un mes previo a la consulta por hiperhidrosis. Ingresó a la unidad de urgencias por un cuadro de cinco días de dolor torácico intermitente, tipo punzada, de intensidad moderada, irradiado a su mandíbula y miembro superior izquierdo, exacerbado a la inspiración y al toser. Asociado, presenta fiebre subjetiva.

Ingresa en mal estado general, febril $\left(39,5{ }^{\circ} \mathrm{C}\right)$, taquicárdica, desaturada (80\%) al ambiente y con signos de dificultad respiratoria. En la auscultación pulmonar se detectó una disminución de los ruidos respiratorios en el hemitórax izquierdo con predominio en el vértice. 
Se le tomaron exámenes paraclínicos que reportaron hemograma con leucocitosis, sin anemia, sin presencia de células inmaduras. Tenía el ácido úrico elevado. El frotis de sangre periférica, las bilirrubinas, los electrolitos y las pruebas de función hepática y renal estuvieron dentro de límites normales. La evaluación cardiológica fue normal con ecocardiograma y con electrocardiograma. Fueron no reactivos el estudio infeccioso con antígeno de superficie de hepatitis B, anticuerpos de hepatitis C y VIH. La prueba tuberculínica (PPD) dio $0 \mathrm{~mm}$.

Se le tomó una radiografía de tórax donde se observó una masa homogénea en el mediastino anterior y proyectado sobre el tercio superior del hemitórax izquierdo, de contornos bien definidos, que mide aproximadamente $101 \times 93$ $\mathrm{mm}$. La silueta cardiaca fue normal (figura 1 ).

Figura 1.Radiografía de tórax con evidencia de imagen radiopaca en el vértice izquierdo

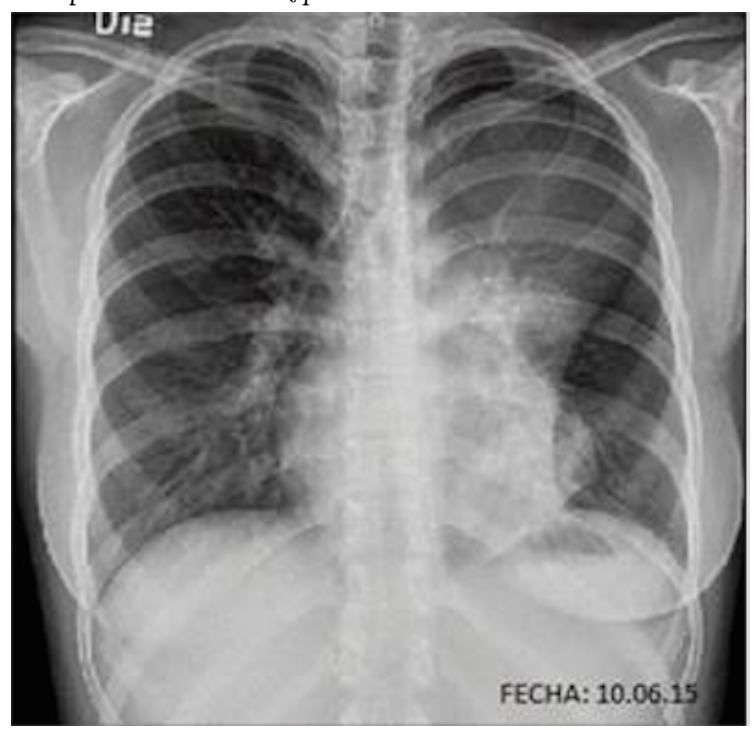

Se indicó que le tomaran una tomografía axial computarizada (TAC) de tórax, donde se observó una masa de $17 \mathrm{~mm}$ en el interior de la luz distal del bronquio fuente izquierdo con obstrucción de la luz para el lóbulo superior (LS). Se evidenció consolidación y pérdida de volumen del lóbulo superior izquierdo, hiperinflación y atelectasia parcial del lóbulo inferior izquierdo y nódulos centrilobulillares en los segmentos basal posterior y lateral del lóbulo inferior izquierdo.
Los anteriores hallazgos eran compatibles con tumor endobronquial (figura 2).

Figura 2.TAC de tórax: masa de $17 \mathrm{~mm}$ en el interior de la luz distal del bronquio fuente izquierdo

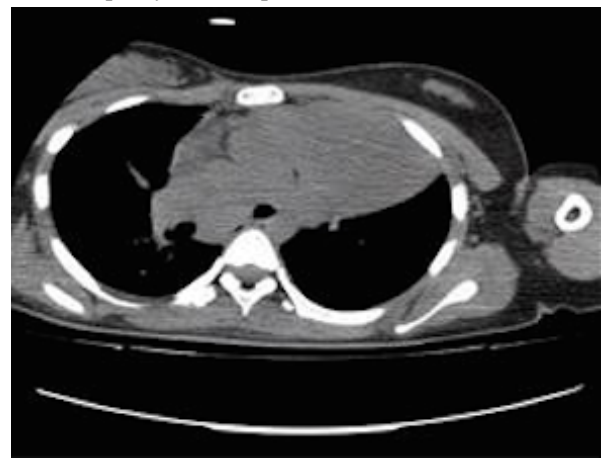

Se le realizó una biopsia percutánea guiada por ecografía, con reporte de patología compatible con proceso neumónico agudo, negativo para malignidad. La adolescente fue llevada a broncoscopia, donde se documentó una oclusión total del bronquio del lóbulo superior izquierdo a $2 \mathrm{~cm}$ de la carina por lesión rojiza compatible con tumor endobronquial (figura 3). Tuvo múltiples biopsias de la lesión y lavado broncoalveolar.

Figura 3.Fibrobroncoscopia: oclusión del $100 \%$ del bronquio fuente izquierdo

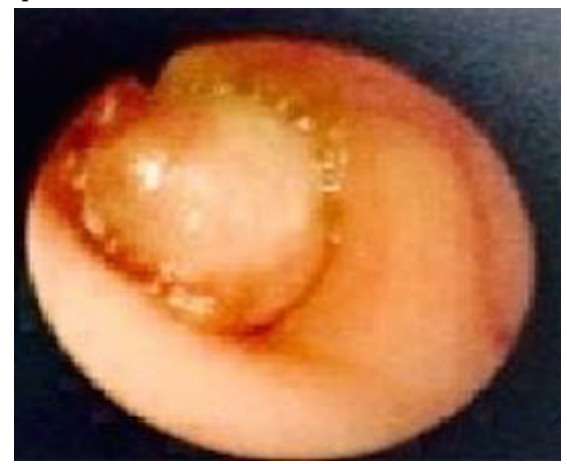

Hubo un reporte de lavado broncoalveolar negativo para malignidad, negativo para gérmenes comunes, con hallazgos compatibles con proceso inflamatorio agudo. La biopsia también reportó cambios de metaplasia escamosa con nidos de células tumorales pequeñas, homogéneas, con cromatinas finas de aspecto en "sal y pimienta", sin mitosis ni necrosis. Los estudios de inmunohistoquímica evidenciaron positividad de las células tumorales para CD56 y cromogranina, con lo que se confirma la 
estirpe neuroendocrina de la lesión, así como su comportamiento biológico de bajo grado con diagnóstico de tumor carcinoide típico (carcinoma neuroendocrino bien diferenciado de bajo grado).

Los resultados de los análisis se llevaron a junta médico-quirúrgica: neumología pediátricaadultos, oncología adultos y cirugía de tórax. Allí se consideró que la paciente se beneficiaría con una resección quirúrgica más exploración endobronquial, y dependiendo de los puntos de clivaje, se haría un manejo definitivo con neumonectomía.

Como imágenes prequirúrgicas se tomó una gammagrafía pulmonar de perfusión. Esta evidenció un aporte del pulmón izquierdo de solamente el $15,82 \%$ de la función. La joven fue llevada a cirugía, donde se le realizó una lobectomía superior izquierda, resección del bronquio fuente izquierdo, broncoplastia izquierda y vaciamiento ganglionar por toracotomía. Luego de ello presentó una evolución posquirúrgica favorable, por lo que se otorga alta con controles.

\section{Discusión}

Los tumores pulmonares primarios, especialmente los tumores endobronquiales, son raros en la infancia, y las series reportadas en la literatura incluyen solo un pequeño número de pacientes [1]. En ausencia de datos prospectivos suficientes, es difícil estimar la incidencia de tumores endobronquiales; sin embargo, los tumores pulmonares constituyen el 0,2\% de todos los casos de neoplasias infantiles [2]. Los tumores pulmonares incluyen tanto las lesiones malignas como los adenomas bronquiales, los carcinoides, los carcinomas mucoepidermoides, el blastoma pleuropulmonar, los carcinomas adenoides quísticos y los tumores benignos [3].

El tumor carcinoide bronquial, por otro lado, es el tumor pulmonar más frecuente en la infancia. Hasta el $75 \%$ de los tumores pulmonares en el niño son malignos; de estos, hasta el $50 \%$ está constituido por los adenomas. El tumor carcinoide supone el $90 \%$ de los adenomas [4].
Los tumores carcinoides son tumores neuroendocrinos que aun cuando con mayor frecuencia se presentan en el tubo digestivo (especialmente en el apéndice), pueden darse en otras localizaciones como el pulmón [5]. Este tipo de neoplasias tiene su origen en las células argentafines asociadas al sistema de captación y descarboxilación de los precursores aminados [6]. En el pulmón se originan en las células de la capa basal del epitelio bronquial [5].

Epidemiológicamente, son tumores que en el pulmón se dan con mayor frecuencia hacia la quinta década de la vida, aunque se pueden dar en otros grupos etarios, como ocurre en el presente caso. Es, además, un tumor clásicamente descrito de predominancia femenina en proporción de tres a uno en el paciente pediátrico [6].

De acuerdo con su ubicación, este tipo de cáncer se clasifica como central y periférico, y según su histología, como típico y atípico. Aquellos centrales se localizan en las paredes de las vías respiratorias grandes; mientras que los periféricos se ubican en la proximidad de la pleura [7].

Según los criterios histológicos revisados por Travis [8], en 1998, se dividen en tumores carcinoides típicos y atípicos, con una frecuencia de $4: 1$, respectivamente. Se determina si son típicos o atípicos según el número de mitosis y la presencia de necrosis. Es importante anotar que los tumores típicos son de crecimiento lento, tienen mayor diferenciación, menor agresividad y rara actividad metastásica. Los atípicos muestran un comportamiento inverso [7].

La Organización Mundial de la Salud estableció en el 2004 una nueva clasificación de los tumores neuroendocrinos pulmonares, al dividirlos en tumores carcinoides típicos $(<2$ mitosis/2 $\mathrm{mm} 2$ con ausencia de necrosis y más de $0,5 \mathrm{~cm}$ ) y tumores carcinoides atípicos (de 2-10 mitosis/2 mm2 o necrosis a menudo punteada). En la nueva clasificación se incluye, además, el tumor neuroendocrino de células grandes y el tumor de células pequeñas [9].

Dada su capacidad de producción de hormonas, como insulina, gastrina, serotonina, péptido intestinal vasoactivo e histamina, estos 
cánceres pueden inducir síntomas sistémicos. Tal es el caso de aquellos tumores productores de serotonina que, al circular por el torrente sanguíneo, son responsables del síndrome carcinoide, se dan en menos del $1 \%$ de los pacientes, y consisten en episodios de rubicundez, sibilancias y diarrea. En otros casos, se puede documentar la presencia de dichas hormonas en la sangre o de sus precursores, como lo es el ácido 5-hidroxiindolacético (precursor de la serotonina), sin que se den manifestaciones evidentes en la clínica [5].

Los síntomas secundarios a la síntesis de hormonas por parte del tumor no son los más usuales. Los pacientes con tumor carcinoide pulmonar presentan con mayor frecuencia otro tipo de síntomas como neumonía recurrente (que es la presentación más usual), tos, hemoptisis (no tan común en niños como en adultos), sibilancias, cuadros imitadores de asma o dolor torácico [5]. Radiológicamente, pueden presentarse como atelectasias [6].

Con la clínica descrita, los diagnósticos diferenciales son amplios, aun cuando son más frecuentes otras entidades, por lo que el diagnóstico de los carcinomas broncogénicos suele ser tardío. En promedio, se logra después de un año de estudios en el contexto de un paciente respiratorio crónico [7] (cuadro 1).

Cuadro 1.Diagnósticos diferenciales del tumor carcinoide bronquial

\begin{tabular}{|l|}
\hline $\begin{array}{l}\text { Procesos infecciosos a } \\
\text { repetición }\end{array}$ \\
\hline Malformaciones \\
\hline $\begin{array}{l}\text { Lesiones } \\
\text { seudotumorales }\end{array}$ \\
\hline $\begin{array}{l}\text { Aspiración de cuerpo } \\
\text { extraño }\end{array}$ \\
\hline Artefactos radiológicos \\
\hline
\end{tabular}

El diagnóstico final se hace mediante un estudio histopatológico con toma de muestra por broncoscopia o cirugía y se complementa con inmunohistoquímica y marcadores neuroendocrinos típicos. Cabe notar que hasta un $75 \%$ de estas neoplasias son visibles por broncoscopia [7].
En tumores broncogénicos pulmonares se ha descrito que hay enfermedad metastásica entre un $5 \%$ y un $27 \%$ de los casos. Es mayor en comparación con la tasa de metástasis de los tumores carcinoides apendiculares, que es muy baja. Se dan principalmente siembras tumorales a ganglios mediastinales, hígado, huesos y piel [6].

Para la detección de metástasis, está descrito el uso de imágenes diagnósticas con somatostatina como marcador (OctreoScan), que parte de la afinidad de los receptores de las células tumorales a dicha molécula. La arteriografía y la gammagrafía son también técnicas útiles descritas para la detección de estos tumores y su extensión [7].

En cuanto al tratamiento, la resección del tumor y de los ganglios regionales es el método de elección, ya que la resección total del tumor se asocia con un muy buen pronóstico. El papel de la quimioterapia aún no es claro [7].

El pronóstico, al igual que en otros cánceres, está ligado con la histología, el grado de invasión, la localización y la presencia de metástasis [7]. Se estima una tasa de supervivencia mayor al $90 \%$ en pacientes con enfermedad localizada y tumores histológicamente catalogados como típicos.

\section{Conclusiones}

El tumor carcinoide bronquial típico es una etiología infrecuente, la cual, si de diagnostica a tiempo, con la resección total de la lesión se llega a una tasa de curación alta; por lo anterior, si realizamos un diagnóstico acertado y oportuno, disminuiremos el riesgo de las complicaciones y la insuficiencia pulmonar que pueden tener los pacientes.

\section{Referencias}

1. Madafferi S, Catania VD, Accinni A, Boldrini $\mathrm{R}$, Inserra A. Endobronchial tumor in children: Unusual finding in recurrent pneumonia, report of three cases. World J Clin Pediatr. 2015;4(2):30-4. 
2. Dishop MK, Kuruvilla S. Primary and metastatic lung tumors in the pediatric population: A review and 25 year experience at a large children's hospital. Arch Pathol Lab Med. 2008;132(7):1079-103.

3. Al-Qahtani AR, Di Lorenzo M, Yazbeck S. Endobronchial tumors in children: Institutional experience and literature review. J Pediatr Surg. 2003;38(5):733-6.

4. Hancock BJ, Di Lorenzo M, Youssef S, Yazbeck S, Marcotte JE, Collin PP. Childhood primary pulmonary neoplasms. J Pediatr Surg. 1993;28(9):1133-6.

5. Puczynski $\mathrm{M}$, Jasty $\mathrm{R}$, Kheralla $\mathrm{N}$, Liang L, Phillipi C, Christensen L, et al. Index of suspicion. Pediatrics in review/American Academy of Pediatrics. 2005;26(8):295-301.

6. Encinas JL, Ávila LF, García-Cabeza MA, Luis A, Hernández F, Martínez L, et al. Tumor carcinoide bronquial y apendicular. Anales de Pediatría. 2006;64(5):474-7.

7. Bisero E, Luque G, Lombardero L, Picón A. Tumor carcinoide pulmonar: A propósito de un caso. Rev Am Med Respirator [internet]. 2014;14(3):318-22. Disponible en: http://www.scielo.org.ar/pdf/ramer/v14 n3/v14n3a17.pdf

8. Pusceddu S, Catena L, Valente M, Buzzoni $\mathrm{R}$, Formisano B, Del Vecchio M, et al. Long-term follow up of patients affected by pulmonary carcinoid at the Istituto Nazionale Tumori of Milan: a retrospective analysis. J Thorac Dis. 2010;2(1):16-20.

9. Madrid-Carbajal C, García-Clemente $M$, Pando-Sandoval A, Cubillas Martín H, González-Budino T, Casán-Clara P. [Bronchial carcinoid tumor: study of 60 patients]. Med Clín. 2013;141(2):73-6. 